OPEN ACCESS

Edited by:

Shuhao Huo

Jiangsu University, China

Reviewed by:

Xupeng Cao,

Dalian Institute of Chemical Physics, Chinese Academy of Sciences (CAS),

China

Changhong Yao

Sichuan University, China

*Correspondence:

Hui Wang

wanghui@qibebt.ac.cn

${ }^{\dagger}$ These authors have contributed equally to this work

Specialty section: This article was submitted to Marine Fisheries, Aquaculture and Living Resources, a section of the journal

Frontiers in Marine Science

Received: 15 December 2021 Accepted: 29 December 2021 Published: 09 February 2022

Citation:

Yin H-C, Sui J-K, Han T-L, Liu T-Z and Wang $H$ (2022) Integration

Bioprocess of B-Phycoerythrin and Exopolysaccharides Production From Photosynthetic Microalga

Porphyridium cruentum.

Front. Mar. Sci. 8:836370.

doi: 10.3389/fmars.2021.836370

\section{Integration Bioprocess of B-Phycoerythrin and Exopolysaccharides Production From Photosynthetic Microalga Porphyridium cruentum}

\author{
Hao-Chan Yin ${ }^{1 \dagger}$, Ji-Kang Sui ${ }^{1+}$, Tian-Li Han 1,2, Tian-Zhong Liu' and Hui Wang ${ }^{1 *}$ \\ ${ }^{1}$ Key Laboratory of Biofuels, Qingdao Institute of Bioenergy and Bioprocess Technology, Chinese Academy of Sciences \\ (CAS), Qingdao, China, ${ }^{2}$ School of Chemical Engineering, University of Chinese Academy of Sciences, Beijing, China
}

Red microalga Porphyridium cruentum has great potential for converting $\mathrm{CO}_{2}$ into high-value bioactive compounds, such as B-phycoerythrin (B-PE) and extracellular polysaccharides or exopolysaccharides (EPS). This study aimed to establish the integration bioprocess of B-PE and EPS production from P. cruentum. First, different kinds of growth medium and $\mathrm{CO}_{2}$ concentration were assessed indoor in terms of high biomass and B-PE and EPS contents. As follows, P. cruentum cells were outdoor scaleup cultured in $700 \mathrm{~L}$ pressurized tubular reactors for 9 days till the biomass reached $0.85 \mathrm{~g} / \mathrm{L}$ and then separated from supernatants via centrifugation. Three different methods were adopted to extract phycobiliproteins, and the highest PE contents were extracted from cells by repeated freeze-thawing treatment along with the optimization of significant variables, and finally, $7.99 \mathrm{mg} / \mathrm{L} \mathrm{B-PE}(16,500 \mathrm{Da})$ with a purity index of 0.82 was obtained. Moreover, analysis of physicochemical properties of EPS extracted from P. cruentum showed that the sulfate content was $14.85 \%$ and the uronic acid content was $9.36 \%$.

Keywords: Porphyridium, biomass production, phycoerythrin, exopolysaccharide, outdoor scale-up culture

\section{INTRODUCTION}

Microalgae are photoautotrophic microorganisms that use light energy, $\mathrm{CO}_{2}$, and inorganic nutrients to synthesize valuable biomass compounds. These photosynthetic microorganisms have the positive advantages of high growth rate and high photosynthetic rate, and their capacity to fix carbon dioxide is 10 times that of terrestrial plants (Lalibertè and Noüie, 2010). In addition, because of the abundant active products, microalga is a good raw material for the production of biofuel and is widely used in food nutraceutical, pharmaceutical, and cosmetic industries as an antioxidant, antimicrobial, and anti-inflammatory product (Li et al., 2008; Francisco et al., 2010; John et al., 2011; Markou and Georgakakis, 2011; Zhu et al., 2016).

Porphyridium cruentum (Rhodophyta, Porphyridiophyceae, Porphyridiales, Porphyridiaceae, Prophyridium) is a spherical unicellular red alga without an organized cell wall (Arad et al., 1985; Adda et al., 1986; Vonshak, 1988; Arad and Levy-Ontman, 2010). This microalga is 
photoautotrophic and can capture light and $\mathrm{CO}_{2}$ into cells to convert them into abundant active molecules. And among these molecules, high contents of B-phycoerythrin (B-PE) and extracellular polysaccharides or exopolysaccharides (EPS) attract more attention. PE is one of the fluorescent substances that host photosynthesis, which shows maximal absorbance at the wavelength range 540-570 nm (Bermejo Román et al., 2002). PE has the advantages of high fluorescence intensity, antioxidation, scavenging free radicals, and high chroma. Thus, it has a wide range of commercial value in food (Fuentes et al., 2000; Gonzalez-Ramirez et al., 2014; Bueno et al., 2020), cosmetics, and pharmaceutical industries and is used as a fluorescent marker, which is dependent on its purity (Koller et al., 1977; Ayyagari et al., 1995; Qiu et al., 2004). Many methods, such as ultrastructure, salting out, column chromatography, and aqueous two-phase system, have been developed for the recovery and purification of B-PE ( Breccia et al., 1998; Romána et al., 2002; Benavides and Rito-Palomares, 2004; Parmar et al., 2011).

Furthermore, Porphyridium polysaccharides are another highvalue compound, which is wrapped on the cell surface in the form of sulfated polysaccharides. In the liquid culture, the tropical part of polysaccharides is dissolved from the cell surface into the medium (Arad and Levy-Ontman, 2010). The average molecular weight of EPS from $P$. cruentum ranges from $1.4 \times 10^{6} \mathrm{Da}$ to $1.7 \times 10^{6} \mathrm{Da}$, and EPS is negatively charged due to the presence of glucuronic acid and sulfate groups (Bernaerts et al., 2018). In addition, EPS is composed of numerous monosaccharides, the abundant glucose, and lactose's characters endow Porphyridium polysaccharide had antiviral and antioxidant activities and effectively inhibit the replication and cell transformation of some retroviruses (Dvir et al., 2000; Talyshinsky et al., 2002).

However, large-scale cultivation and commercial applications of $P$. cruentum have not achieved extensive use. To our knowledge, there is a crucial problem about how to promote the biomass and the yield of B-PE and EPS for large-scale cultivation (Spolaore et al., 2006). Moreover, despite the purification methods of B-PE and EPS from P. cruentum being developed, respectively, in previous studies, the joint extraction process of B-PE and EPS from P. cruentum culture is still rare, especially on a large scale. Therefore, in this study, microalga $P$. cruentum was initially cultured indoors with different mediums, and $\mathrm{CO}_{2}$ concentration was considered as a key parameter to assess the accumulation of biomass, PE, and EPS production. As follows, outdoor scale cultivation with $700 \mathrm{~L}$ was carried out with optimal medium and $\mathrm{CO}_{2}$ supply to obtain a large amount of biomass. After centrifugation, PE from biomass and EPS from culture were extracted and their basic performance analysis was investigated. The results will supply integration and optimal strategy for the customized production of B-PE and EPS from P. cruentum.

\section{MATERIALS AND METHODS}

\section{Subsection Microalgal Growth Conditions Indoor}

Photoautotrophic red microalga $P$. cruentum was maintained in $30 \mathrm{ml}$ of $\mathrm{f} / 2$ medium in $100 \mathrm{ml}$ Erlenmeyer flasks without aeration. Light at an intensity of $100 \mu \mathrm{mol}$ photon $/ \mathrm{m}^{2} \mathrm{~s}$ was continuously supplied with $\mathrm{pH}$ adjusted at 7.6 and temperature at $25 \pm{ }^{\circ} \mathrm{C}$. Continuous shaking of the culture flasks was done, 2-3 times a day, to prevent sticking of the culture to the bottom of the flask.

\section{Subsection Microalgal Growth With Different Conditions Indoor}

For the medium treatment experiment, starter cultures in the logarithmic phase were gently centrifuged (5,000 rpm for $10 \mathrm{~min}$ ) and washed with sterile distilled water. The microalgal pellets were resuspended in a small volume of sterile distilled water. The suspensions were then used to inoculate different mediums $(700 \mathrm{ml})$ present in a glass column photobioreactor (PBR): artificial seawater (ASW) (Jones et al., 2010), BG11-seawater (SBG11) (Liu et al., 2013), f/2 (Guillard and Ryther, 1962), and $4 f$ $(8 \times \mathrm{f} / 2)$. Illumination was provided by single-side fluorescent lamps, which produced $100 \mu \mathrm{mol}$ photon $/ \mathrm{m}^{2} \mathrm{~s}$. The carbon source and agitation were supplied by bubbling $\mathrm{CO}_{2}$-enriched compressed air $\left(1 \% \mathrm{CO}_{2}, \mathrm{v} / \mathrm{v}\right)$, which was filtered through the disposable sterile filter.

At the base optimal medium, cultures $(700 \mathrm{ml})$ of $P$. cruentum were grown in the optimal medium with $100 \mu \mathrm{mol}$ photon $/ \mathrm{m}^{2} \mathrm{~s}$, and compressed air containing different levels of $\mathrm{CO}_{2}(0.04 \%$, $1 \%, 3 \%$, and $5 \%$ ) was aerated continuously into the cultivation medium. The concentration of the starting biomass was ca. $0.2 \mathrm{~g} / \mathrm{L}$, and all the experiments were carried out at $25 \pm 1^{\circ} \mathrm{C}$.

\section{Growth and Active Compounds Accumulation}

Microalgal dry weight (DW) was measured according to a method previously reported (Talyshinsky et al., 2002). A certain volume of microalgal culture was filtered to a pre-weighted $0.45 \mu \mathrm{m}$ GF/C filter membrane (Whatman, DW0). The membrane was oven-dried at $105^{\circ} \mathrm{C}$ overnight and then weighted (DW1). The DW was calculated as:

$$
\mathrm{DW}=\frac{D W_{1}-D W_{0}}{\mathrm{~V}}
$$

For the determination of EPS concentration, a $10 \mathrm{ml}$ aliquot of microalgal culture was originally centrifuged at $8,000 \mathrm{rpm}$ for $10 \mathrm{~min}$. The supernatant was collected and stored at $-4^{\circ} \mathrm{C}$ for the determination of EPS concentration. The EPS content was measured using the phenol-sulfuric acid method as described by Dubois et al. (1956).

Freeze-dried biomass was used to determine the PE content. The phycobiliproteins were extracted with $0.1 \mathrm{M}$ phosphate buffer ( $\mathrm{pH}$ 6.8) using a freeze-thawing technique as described below (Bermejo et al., 2003). Repeatedly extract the cells until the algae residue has no obvious red color and combine the extracts to a final volume of $50 \mathrm{ml}$. The absorbance of the solution was measured at 455, 564, and $592 \mathrm{~nm}$ with an ultraviolet spectrophotometer. The content of $\mathrm{PE}$ is calculated according to the formula used in the method of Beer and Eshel (1985). 


\section{Large Cultivation of Microalgal Culture Outdoor}

At the basis of indoor cultivation, microalga $P$. cruentum was cultivated in a $700 \mathrm{~L}$ pressurized tubular PBR with optimal medium and $\mathrm{CO}_{2}$ supply. The PBR was set outdoor, and the intensity of natural light was set using curtains. The $\mathrm{pH}$ set point of 7.6 was automatically controlled by $\mathrm{CO}_{2}$ injection (3\%) and monitored by an inline Fermprobe $\mathrm{pH}$ probe. Air was injected continuously at a rate of $35 \mathrm{~L} / \mathrm{min}$. After 10 days, cells were harvested by bowl centrifugation at $6,000 \mathrm{rpm}$ at room temperature and concentrated at a rate of ca. $10 \%$ dry weight. The biomass was then dispatched in aliquots in airtight glass bottles at $-20^{\circ} \mathrm{C}$ away from light. And the supernatants were kept in the plastic drum at cold storage.

\section{Phycobiliproteins Extraction}

Phycobiliprotein crude exact was obtained from $P$. cruentum with different methods:

1) Repeated freezing and thawing treatment: Suspend the microalgal particles and wash them with $18 \mathrm{M} \Omega$ Milli$\mathrm{Q}$ pure water, then centrifuge for $10 \mathrm{~min}(12,000 \times \mathrm{g}$, $T=10^{\circ} \mathrm{C}$ ), and remove the supernatant. The pellet was suspended in $10 \mathrm{ml}$ of extracting solution [phosphatebuffered saline (PBS): $10 \mathrm{mM}$ phosphate, $100 \mathrm{mM} \mathrm{NaCl}$, pH7] and was freeze-thawed 3 times between $-20^{\circ} \mathrm{C}$ and room temperature $\left(20-25^{\circ} \mathrm{C}\right)$. After repeating freezethawing treatments for 3 times, the suspension was kept at $4^{\circ} \mathrm{C}$ for $24 \mathrm{~h}$ and then centrifuged for $10 \mathrm{~min}$ $\left(12,000 \times g, T=10^{\circ} \mathrm{C}\right)$. Collect the supernatant containing phycobiliprotein (crude extract) and store it at $4^{\circ} \mathrm{C}$ (Bermejo et al., 2003).

2) Liquid nitrogen grinding treatment: Proper amount of microalgal biomass was loaded into a mortar and mixed with the appropriate amount of liquid nitrogen and ground into powder, during which the liquid nitrogen is continuously loaded. Deionized water was added to dissolve the power, and the suspension was finally centrifuged for $10 \mathrm{~min}\left(12,000 \times g, T=10^{\circ} \mathrm{C}\right)$ to collect the supernatant (Chopin et al., 2000).

3) Sonication treatment with bead milling: This method was used as described by Zheng et al. (2011). The proper amount of microalgal biomass was dissolved in deionized water, and then a small steel ball with $3 \mathrm{~mm}$ diameter was added. Then, the mixture was sonicated four times for $60 \mathrm{~s}$ in a water/ice bath, with a $60 \mathrm{~s}$ pause in-between (to avoid phycobiliprotein degradation). The suspension was finally centrifuged, and the supernatant containing the phycobiliproteins (crude extract) was collected and stored at $4^{\circ} \mathrm{C}$.

\section{Phycoerythrin Purification and Identification}

In this study, we used algal mud or powder for extraction; accordingly, $10 \mathrm{ml}$ of $18 \mathrm{M} \Omega$ Milli-Q pure water was added for $0.3 \mathrm{~g}$. The method of Romána et al. (2002) was used to purify the crude PE extract. First, ammonium sulfate was added to the aqueous phycobiliproteins extract to make the saturation reach $20 \%$ for $3 \mathrm{~h}$, and then, the mixture was centrifuged at $8,500 \times g$ for $10 \mathrm{~min}$ to remove impurities. Next, ammonium sulfate was added to reach $60 \%$ saturation overnight at $4^{\circ} \mathrm{C}$ and then centrifuged again to obtain PE precipitate. Then, the target $\mathrm{PE}$ was re-dissolved in a small amount of ionized water and stored at $4^{\circ} \mathrm{C}$. The PE content was calculated as described above, and the purity was calculated as the absorbance ratio of 545/280 nm.

Phycoerythrin identification was determined using the SDSPAGE method and spectroscopic methods. For SDS-PAGE, electrophoresis was carried out in a vertical slab gel apparatus (Miniprotean III, Bio-Rad) according to the tricine buffer system described by Schagger and Jagow (1987) using a 16.5\% polyacrylamide slab gel containing $0.1 \%(\mathrm{w} / \mathrm{v})$ SDS with a stacking gel of $4 \%$ polyacrylamide. Samples were pre-incubated with buffer at ratio $4: 1$ at $95^{\circ} \mathrm{C}$ for about $10 \mathrm{~min}$. Gels were run $45 \mathrm{~min}$ with $120 \mathrm{~V}$ constant voltage at room temperature and visualized by staining for $30 \mathrm{~min}$ with $0.1 \%(\mathrm{w} / \mathrm{v})$ Coomassie Brilliant Blue R-250, 40\% methanol (v/v) with 7\% (v/v) acetic acid, and destained in dilute acetic acid. For the spectroscopic method, the PE was fully wavelength scanned.

\section{Exopolysaccharides Extraction and Analysis}

As described above, microalgal culture was centrifuged and the supernatant was collected. A volume of ethanol was added to the supernatant [supernatant:ethanol $=1: 3(\mathrm{v} / \mathrm{v})$ ] and fully mixed overnight, and the temperature was kept at $4^{\circ} \mathrm{C}$. To avoid the disturbance of monosaccharides and oligosaccharides, the supernatant was dialyzed using a dialysis bag of $8 \mathrm{kDa}$. After that, the dialyzed sugar solution was precipitated with ethanol once again. Finally, the EPS was freeze-dried in vacuo.

The physicochemical properties of EPS were determined. First of all, the sulfate content was determined using the barium sulfate turbidity method (Zhang, 2003). The concentration of glucuronic acid was measured using the sulfuric acid-carbazole colorimetry method (Dodgson and Price, 1962). The rheological properties of polysaccharide aqueous solutions were measured using an NDJ$8 \mathrm{~S}$ digital viscometer with a 2 -size rotor (Shanghai, China).

\section{RESULTS}

\section{Growth and Active Compounds Accumulation Indoor}

Microalga $P$. cruentum was cultivated in different mediums for 15 days, and the biomass, PE, and EPS contents were analyzed every 3 days. On the whole, $P$. creuntum showed marked differences in growth and active compounds accumulation in ASW, BG11-seawater, f/2, and 4f culture medium (Figure 1). Under a light intensity of $100 \mu \mathrm{mol}$ photon $/ \mathrm{m}^{2} \mathrm{~s}$, a lag phase of 4 days, 8 days of exponential phase, and 3 days of reduction phase were observed for the four culture mediums evaluated. The lowest biomass production was observed at the $\mathrm{f} / 2$ culture medium, the maximum specific growth rate and productivity 


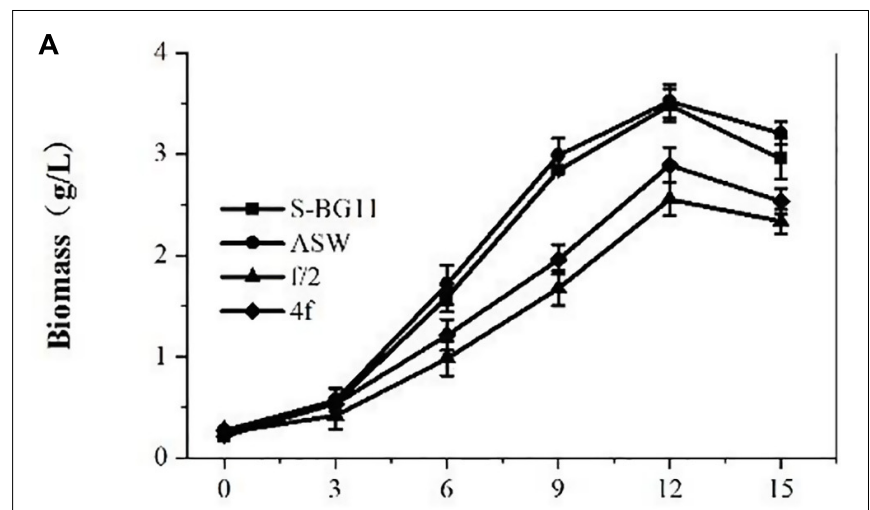

B

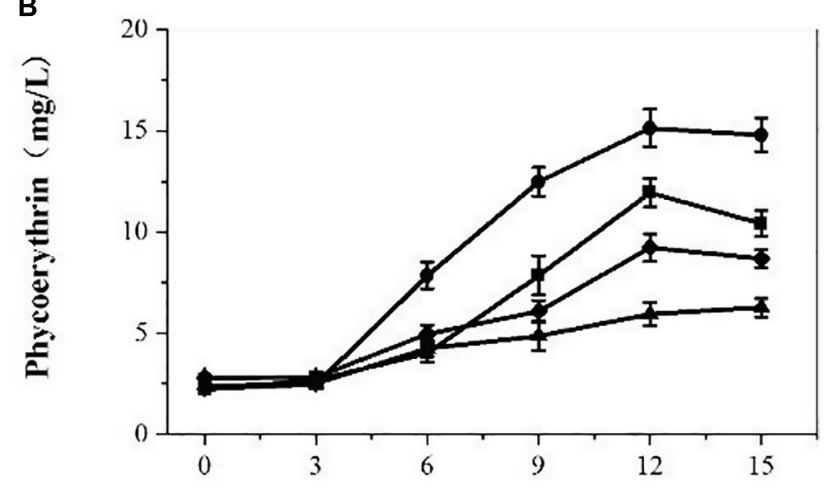

C

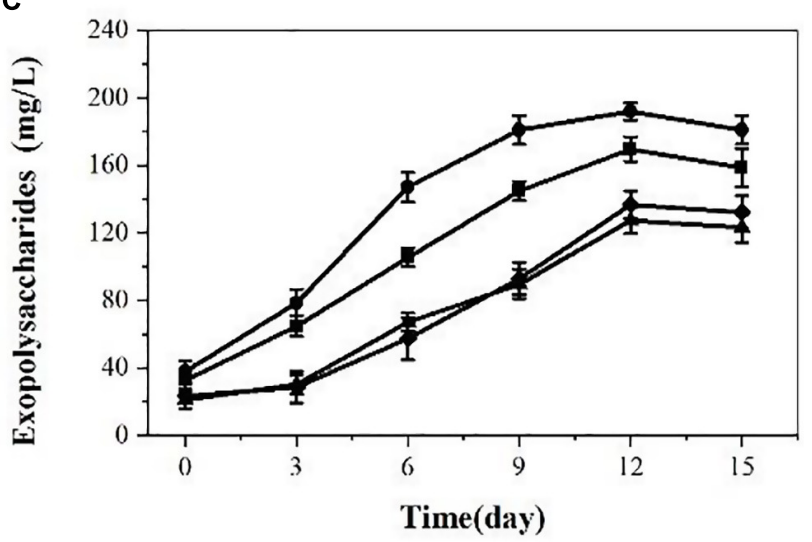

FIGURE 1 | Biomass (A), phycoerythrin (B), and exopolysaccharides (C) contents of $P$. cruentum in different culture mediums.

values were higher for the biomass with a culture medium of ASW, and the multiplication time of microalgal cells was shorter, compared with the condition of the other culture medium. Overall, the culture reached a maximum biomass concentration at day 12 with $3.48,3.52,2.55$, and $2.88 \mathrm{~g} / \mathrm{L}$ for ASW, S-BG11, f/2, and $4 \mathrm{f}$, respectively.

Phycoerythrin production by $P$. cruentum was also analyzed under different culture mediums, and it showed similar lag phases among these culture mediums (Figure 1B). A maximum PE concentration of $15.14 \mathrm{mg} / \mathrm{L}$ was observed in the ASW culture medium on the 12th day. Lower PE content was observed in the f/ 2 culture medium. The EPS production by $P$. cruentum under different culture mediums is presented in Figure 1C. The four culture mediums showed a similar trend, and EPS production increased at the first 12 days of culture and showed a downward trend in the last 3 days. The maximum EPS concentration was observed on the 12th day of culture with $169.72,191.88$, 127.24, $136.67 \mathrm{mg} / \mathrm{L}$, respectively. These could be associated with the fact that the mediums had different nutrient factors and concentrations.

At the base of the optimal medium, we investigated the effects of $\mathrm{CO}_{2}$ concentration on $P$. cruentum growth, PE, and EPS production (Figure 2). The biomass production from the culture of the $P$. cruentum under different $\mathrm{CO}_{2}$ concentrations is presented in Figure 2A. Overall, a lag phase of 3 days was observed, the culture reached a maximum biomass concentration at day 12, and then, the growth inhibited in the last 3 days. When the concentration of $\mathrm{CO}_{2}$ was $3 \%$, it resulted in the maximum biomass production of $4.52 \mathrm{~g} / \mathrm{L}$. The maximal biomass production for treatments with $\mathrm{CO}_{2}$ concentrations of 0.04 , 1 , and 5 were $2.94,3.68$, and $4.24 \mathrm{~g} / \mathrm{L}$, respectively. The PE production by $P$. cruentum under different $\mathrm{CO}_{2}$ concentrations is presented in Figure 2B. A maximum PE concentration of $18.37 \mathrm{mg} / \mathrm{L}$ was observed at $5 \% \mathrm{CO}_{2}$ on the 12 th day of culture. Similarly, $18.17 \mathrm{mg} / \mathrm{L} \mathrm{PE}$ was determined at $3 \% \mathrm{CO}_{2}$ on the 12th day. The PE content was lower when the concentration of $\mathrm{CO}_{2}$ was $0.04 \%$ in the first 6 days. Similar to the biomass, the highest values of $\mathrm{PE}$ content were observed under $3 \% \mathrm{CO}_{2}$ concentration. The results of EPS concentration under different $\mathrm{CO}_{2}$ concentrations are shown in Figure 2C. Overall, the culture reached a maximum EPS concentration at day 12, with 137.17, $152.15,202.39$, and $218.4 \mathrm{mg} / \mathrm{L}$. Furthermore, the highest values of EPS content were obtained under the $\mathrm{CO}_{2}$ concentration of $5 \%$. The high EPS content was determined at $3 \% \mathrm{CO}_{2}$ obtained on the 12th day. The EPS content was lower when the concentration of $\mathrm{CO}_{2}$ was $0.04 \%$. The experiments performed in ASW culture medium at $3 \% \mathrm{CO}_{2}$ concentration resulted in a maximum $\mathrm{PE}$ and biomass production after 12 days.

\section{Growth and Active Compounds Accumulation Outdoor}

On the above results indoor, we cultivated P. cruentum in ASW medium in the $700 \mathrm{~L}$ pressurized tubular PBR and offered compressed air with $3 \% \mathrm{CO}_{2}$ outdoor. The biomass and EPS production of $P$. cruentum were continuously monitored during the cultivation period. Five days of lag stage and 4 days of growth stage were observed (Figure 3B). P. cruentum reached its maximum biomass production of $0.85 \mathrm{~g} / \mathrm{L}$ on the 9 th day of culture. During the last 2 days of cultivation, the growth rate reduced and the biomass was inhibited. The EPS concentration for P. cruentum culture in the tubular PBR is shown in Figure 3A; a slow growth phase for 5 days and a stable growth phase were observed. The maximum EPS concentration of $11.41 \mathrm{mg} / \mathrm{L}$ was obtained on the 9th day of culture (Figure 3C). The difference in the results obtained between outdoor and indoor experiments may be due to uncontrollable factors, such as light intensity and temperature and the low inoculation. 


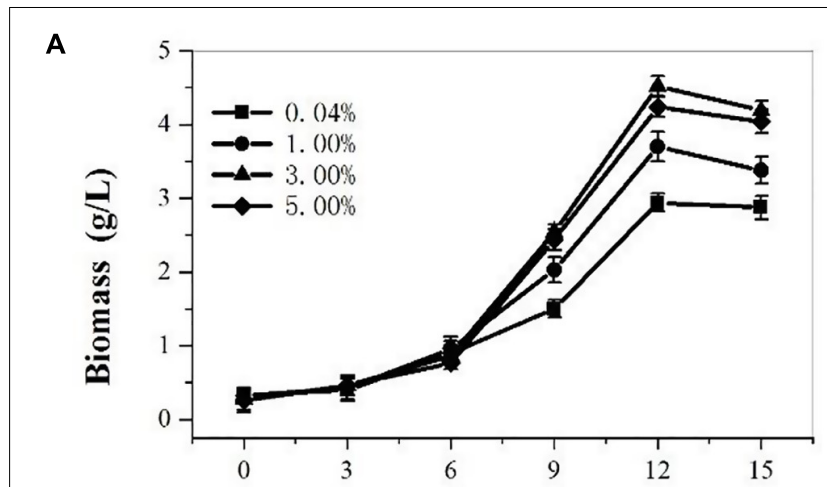

B
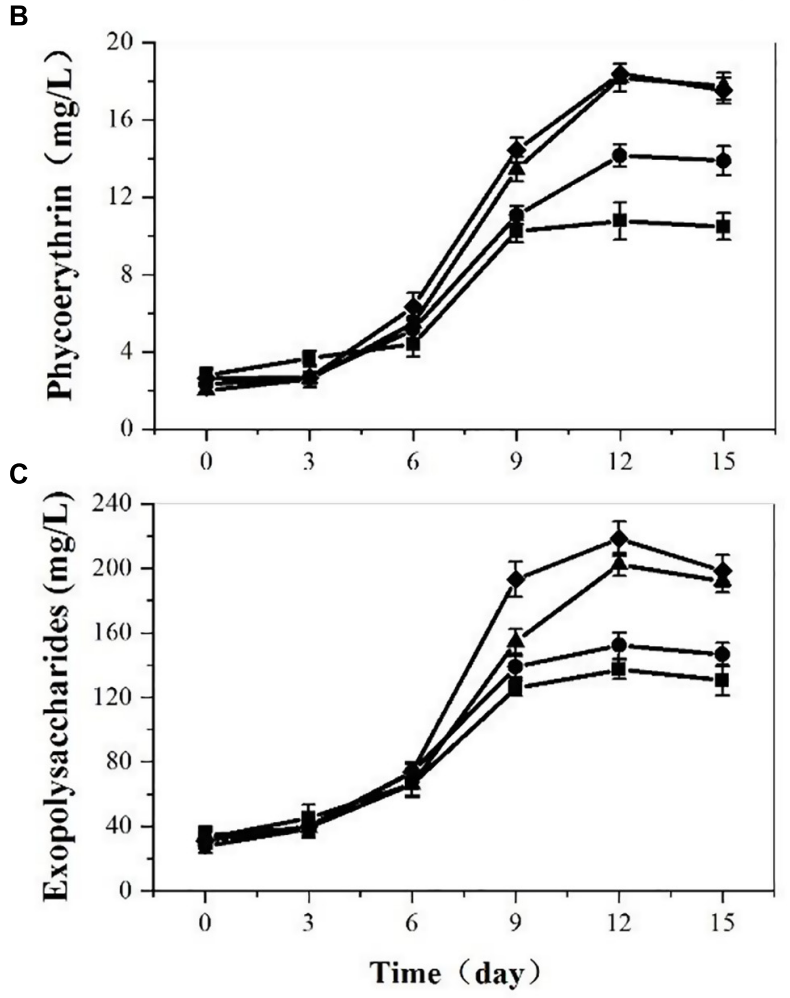

FIGURE 2 | Biomass (A), phycoerythrin (B), and exopolysaccharides (C) contents of Porphyridium cruentum in ASW with different $\mathrm{CO}_{2}$ concentrations.

\section{Extraction of Phycoerythrin via Different Methods}

In this study, we used three different methods to extract PE from P. cruentum cells and measured its concentrations and purity (Figure 4). The concentrations of PE from P. cruentum were 7.99, 5.27 , and $6.34 \mathrm{mg} / \mathrm{L}$ for repeated freeze-thawing treatment, liquid nitrogen grinding, and sonication with bead milling, respectively. From the data, the repeated freeze-thawing treatment, which we adopted in indoor cultivation, could reach the highest extraction efficiency. Being different from the PE extraction concentrations, the three extraction methods did not show significant effects on the purities of PE. As illustrated in Figure 4, the PE purities of $0.82,0.91$, and 0.83 were obtained, respectively, from P. cruentum for the abovementioned three extraction methods.

\section{Analysis of Phycoerythrin Extraction}

Ultraviolet absorption spectra of PE extraction via liquid nitrogen grinding method are presented in Figure 5A. The spectrum of PE displayed two maxima, namely, 545 and $564 \mathrm{~nm}$; and exhibited only a slight shoulder at $499 \mathrm{~nm}$. According to the ultraviolet absorption characteristics, there are mainly two types of $\mathrm{PE}$ in red microalga, namely, B-PE and R-phycoerythrin (R-PE). B-PE exhibits two absorption peaks at 545 and $564 \mathrm{~nm}$, and one absorption shoulder at $499 \mathrm{~nm}$; R-PE exhibits two characteristic absorption peaks at 499 and $564 \mathrm{~nm}$, and one absorption shoulder at $545 \mathrm{~nm}$ (Glazer, 1984; Hilditch et al., 1991; Galland-Irmouli et al., 2000). It can be concluded that the PE fraction extracted and purified in this study is B-PE.

The purification was followed by electrophoretic experiments to confirm it. In this way, the electrophoretic analysis was carried out using the fraction obtained above (Figure 5B). By comparison with standards, we mainly obtained a molecular mass of $16,500 \mathrm{Da}$. It is similar to the band of the PE complex extracted from the marine red alga Griffithsia pacifica (Zhang et al., 2017).

\section{Analysis of Exopolysaccharides}

In this study, we used the ultraviolet spectrum scanning method to evaluate the EPS extraction. According to the examination, there was no obvious characteristic absorption peak at $280 \mathrm{~nm}$; it can be concluded that the crude polysaccharide contains no protein (data not shown). The biological activity of polysaccharides is usually related to their sulfate group and uronic acid content. In this study, the crude polysaccharide was configured into a $1 \mathrm{~g} / \mathrm{L}$ solution for sulfate group and uronic acid content detection. The sulfate group content and the uronic acid content of the crude polysaccharide were $14.85 \%$ and $9.36 \%$, respectively.

The rheological properties of polysaccharides are related to their concentration. The viscosity of the solution increased with the increment of the concentrations of the polysaccharide solution which indicated thickening performance (Figure 6). The polysaccharide solution showed significant shear-thinning behavior; the viscosity of the solution decreased with the increasing shear rate and exhibited the characteristics of pseudoplastic fluids. With the increase in temperature $\left(25-75^{\circ} \mathrm{C}\right)$, the viscosity does not change much, which indicates that the polysaccharide solution has higher heat resistance.

\section{DISCUSSION}

The culture medium can indicate the growth, PE, and EPS accumulation of $P$. cruentum. This study showed that biomass or growth rate of $P$. cruentum could reach its higher value in artificial seawater water (ASW) medium and BG11-seawater (SBG11) (Figure 1A), which is in agreement with the results of the previous study for Prophyridium (Coward et al., 2016). During the cultivation period, the ASW and S-BG11 groups reached up to 3.48 and $3.52 \mathrm{~g} / \mathrm{L}$ of biomass concentration, which was mostly higher than the concentrations reported in previous studies. The maximum biomass concentrations in P. cruentum reported in the studies by Razaghi et al. (2014) and Hu et al. (2018) were 1.61 and 
A

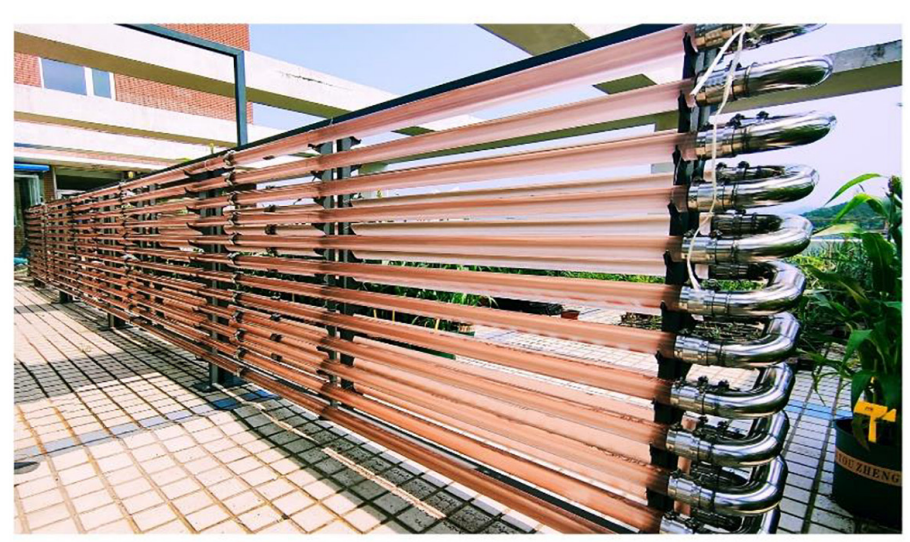

B

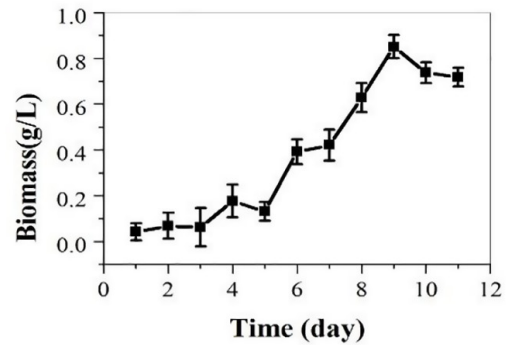

C

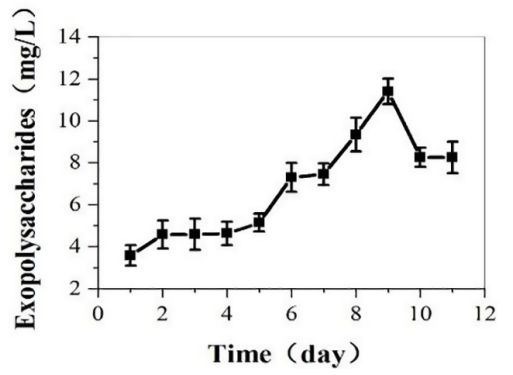

FIGURE 3 | Biomass (B) and exopolysaccharides (C) contents of $P$. cruentum in large-scale cultivation outdoor (A).

$1.22 \mathrm{~g} / \mathrm{L}$, respectively. This may be due to the continuous supply of available carbon during the entire cultivation period of this study. Besides biomass, PE and EPS contents were significantly different among these tested culture mediums due to abundant nutrients. Overall, ASW results in significantly higher contents in PE $(15.14 \mathrm{mg} / \mathrm{L})$ and EPS $(191.88 \mathrm{mg} / \mathrm{L})$ accumulation of P. cruentum than S-BG11 and other mediums. Therefore, ASW

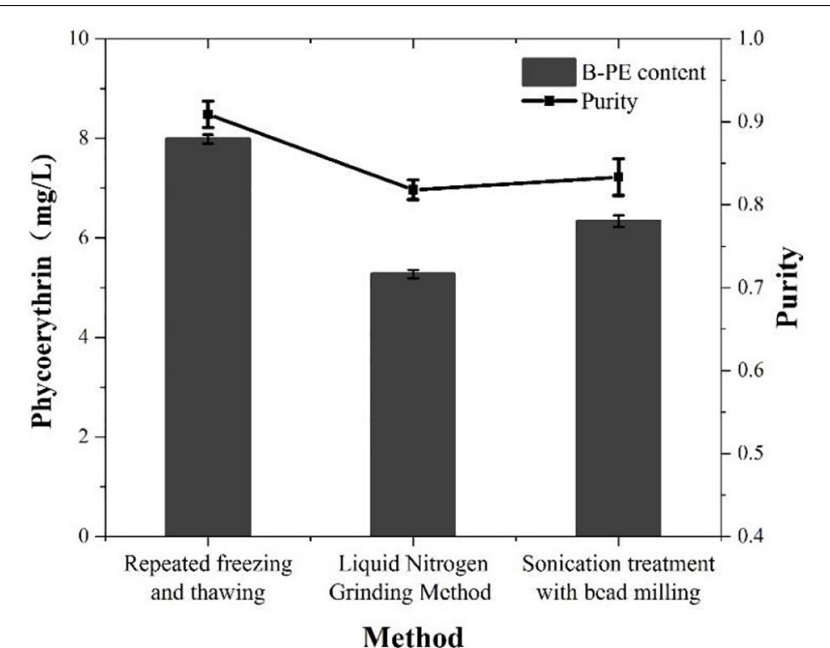

FIGURE 4 | Phycoerythrin contents and purities of Porphyridium cruentum with different extraction methods. as the appropriate culture medium was used further in the following studies.

Besides culture medium, environmental factors, such as temperature, $\mathrm{CO}_{2}$ concentration, and light regime, can directly influence the photosynthetic rate and metabolic activity of the microalga, thereby affecting the biomass and the overall accumulation of active compounds in the microalga. Among these, in scale cultivation outdoor, $\mathrm{CO}_{2}$ concentration could be easily adjustable, while light intensity and temperature are not controllable. Therefore, in this study, the effect of $\mathrm{CO}_{2}$ concentration on biomass and active compounds was also determined. Overall, with the increase of $\mathrm{CO}_{2}$ concentration, the biomass, PE, and EPS contents of $P$. cruentum increased. The highest concentrations of PE $(18.37 \mathrm{mg} / \mathrm{L})$ and EPS $(218.5 \mathrm{mg} / \mathrm{L})$ were achieved with $5 \% \mathrm{CO}_{2}$; however, there was no significant difference in biomass and $\mathrm{PE}$ between $3 \%$ and $5 \% \mathrm{CO}_{2}$.

According to the abovementioned results, P. cruentum was cultivated outdoor in a $700 \mathrm{~L}$ pressurized tubular PBR with $3 \% \mathrm{CO}_{2}$ supply in ASW (Figure 3). In this section, the biomass of $P$. cruentum was significantly lower than those in indoor cultivation, because of the intermittent illumination and uncontrollable temperature outdoor. Light is an essential source for autotrophic growth, and photosynthetic activity in microalga, light intensity, and light regime leads to different changes, such as photo-inhibition and photo-insufficient, even in dense culture and thus can be detrimental to the final yield (Papageorgiou et al., 2007). Moreover, the temperature is another major factor that affects the growth rate and chemical composition of microalga. Previous studies performed with microalgal strains have shown 
A

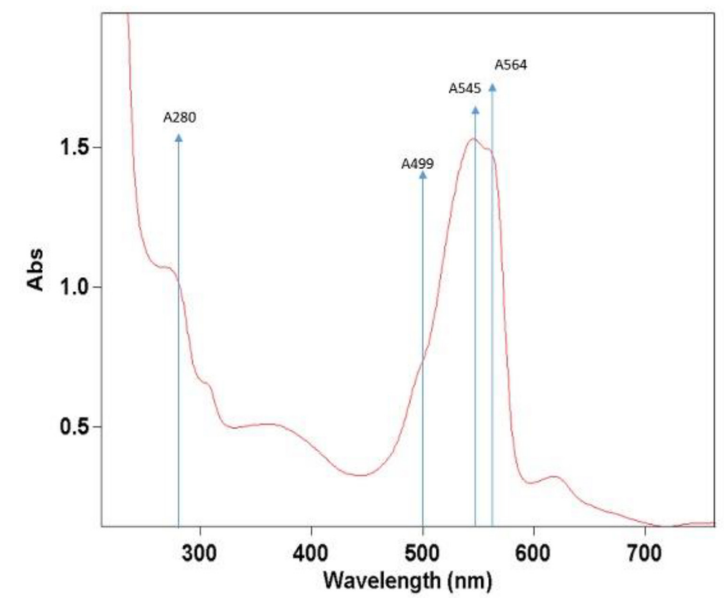

B

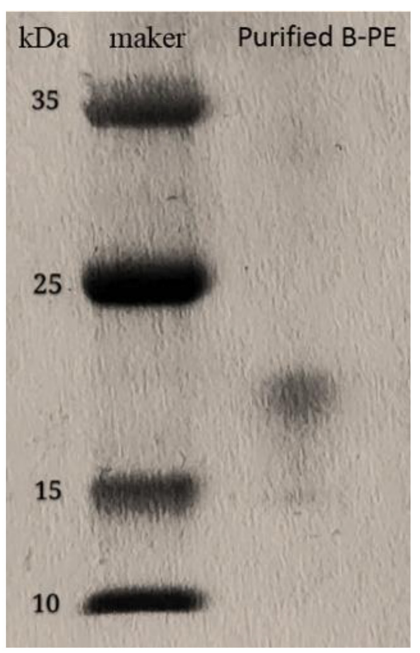

FIGURE 5 | Identification of phycoerythrin of Porphyridium cruentum via ultraviolet absorption spectra (A) and SDS-PAGE method (B).

that the optimal temperature was $18-25^{\circ} \mathrm{C}$ (Srirangan et al., 2015). In outdoor cultivation, the temperature fluctuates up and down and results in biomass reduction. P. cruentum cells secrete EPS, which is considered an optimal ingredient in cosmetics because of its excellent moisturizing properties. Our results

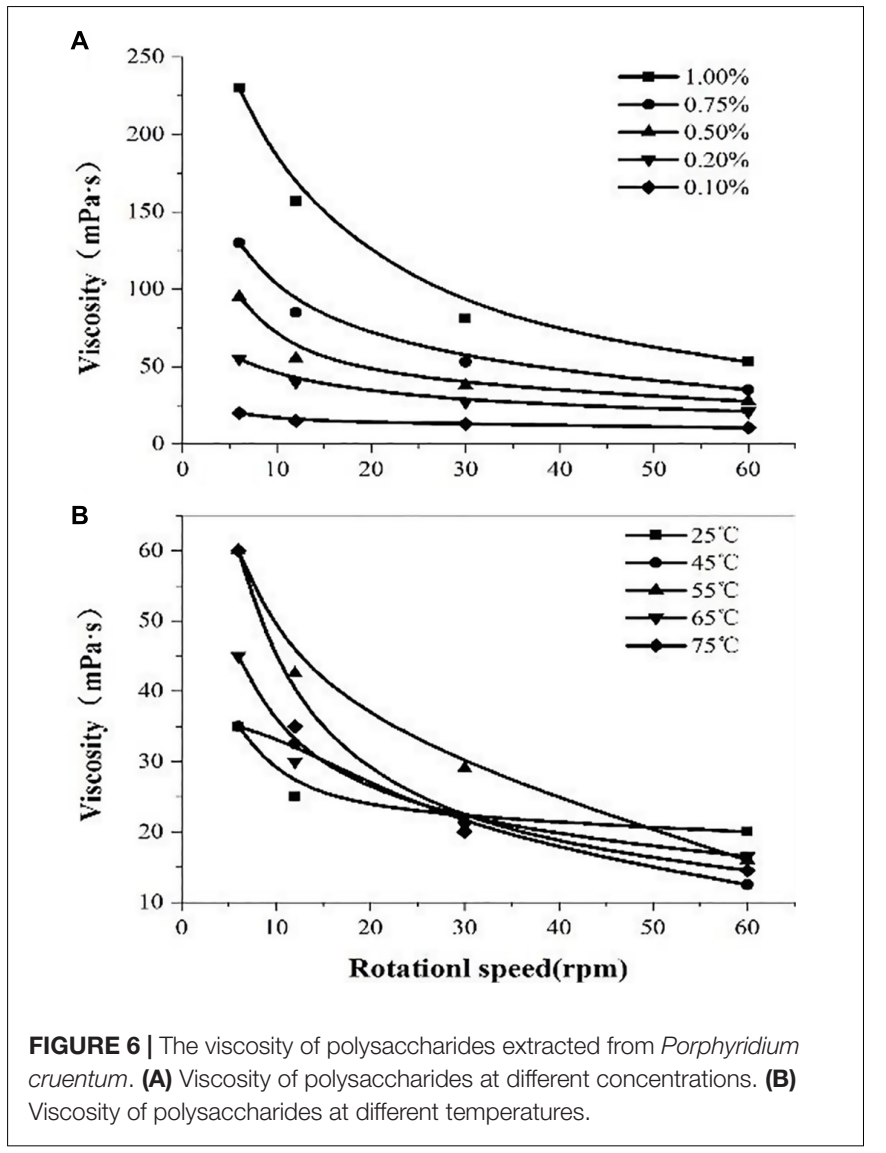

showed that the concentration of EPS gradually increased with the culture time, but the accumulation of EPS indicated a significant difference between indoor and outdoor cultivation because the biomass was reduced outdoor (Figure 3B).

Microalga $P$. cruentum contains three types of phycobiliproteins, namely, B-PE, PC, and APC (Marsac, 2003). Among these, B-PE is one of the most well-known pigment-protein complexes that are involved in photosynthesis in microalgal cells (Gough and Kannangara, 1979), and thus, the $\mathrm{PE}$ accumulation is closely related to the change of light quality (spectra composition), light intensity, and light regime (Barth et al., 2014). Concerning the target production of phycobiliproteins, it has been widely described as a preference for low and medium intensities of light for the production of these pigments (Rizzo et al., 2015). This can be explained by the function of these compounds in the photosynthetic apparatus. However, light intensity outdoor could reach a light intensity higher than 4,000 lux; the phycobiliproteins, like carotenoids, are not required to produce an increase in the range of light absorption.

To obtain target phycobiliproteins, extraction and purification procedures are required. According to the previous study (Mittal et al., 2017), a crude extraction can be done by mechanical disruption of the cell (ultrasonication, bead mill, or high-pressure systems) or even by chemical osmotic extraction. In this study, we used three methods (e.g., repeated freeze-thawing treatment, liquid nitrogen grinding treatment, and sonication treatment with beading milling) to extract the phycobiliprotein crude from microalgal cells that were cultivated outdoor and compared the extraction efficiency and purity of PE. As illustrated in Figure 4, the highest extraction efficiency of PE was obtained via repeated freeze-thawing treatment, while the highest purity of PE was got via liquid nitrogen grinding treatment. The in-depth research and optimization of extraction of phycobiliproteins to get higher extraction efficiency and purity will be studied in the future. Although the most utilized criterion to check the purity of 
biliprotein solutions is absorbance, the purity and identification must be followed by electrophoretic experiments to confirm it. In this way, the spectroscopic and electrophoretic analysis of crude phycobiliprotein obtained from P. cruentum was carried out. Electrophoretic analysis revealed broadband between 15 and $25 \mathrm{kDa}$, which could be assigned to subunits $\alpha$ and $\beta$, which was consistent with the value reported previously (Zhang et al., 2017). Figure 5 shows visible absorption, fluorescence emission, and anisotropy spectra of purified B-PE from P. cruentum. Absorbance maximum is $545 \mathrm{~nm}$, whereas the fluorescence emission maximum is $564 \mathrm{~nm}$ and the anisotropy spectrum is similar to those earlier published for PEs from another red alga (Maccoll, 1991).

In addition, the fundamental characteristics of EPS extracted from $P$. cruentum were evaluated in this study (Figure 6). Former literature introduced that EPS from red alga contains $\beta$-1,3-glycosidic bonds and sulfates, which endow EPS with antiviral, antioxidant, and radical-scavenging functions (Sun et al., 2009). Our results showed that the sulfate content of EPS from $P$. cruentum was $14.85 \%$ and the uronic acid content was $9.36 \%$. Moreover, the polysaccharide solution shows a significant shear-thinning behavior and has higher heat resistance.

\section{CONCLUSION}

It is clear from this study that culture medium, $\mathrm{CO}_{2}$ concentration, and light regime can affect the growth and active compounds accumulation of $P$. cruentum. According to the indoor experiment, optimal medium and $\mathrm{CO}_{2}$ concentration were chosen to cultivate $P$. cruentum in a $700 \mathrm{~L}$ pressurized tubular PBR outdoor. However, uncontrollable factors including

\section{REFERENCES}

Adda, M., Merchuk, J. C., and Arad, S. (1986). Effect of nitrate on growth and production of cell-wall polysaccharide by the unicellular red alga Porphyridium. Biomass 10, 131-140. doi: 10.1016/0144-4565(86)90061-2

Arad, S. M., Adda, M., and Cohen, E. (1985). The potential of production of sulfated polysaccharides from Porphyridium. Plant Soil 89, 117-127.

Arad, S. M., and Levy-Ontman, O. (2010). Red microalgal cell-wall polysaccharides. biotechnological. aspects. Curr. Opin. Biotechnol. 21, 358-364. doi: 10.1016/j.copbio.2010.02.008

Ayyagari, M. S., Pande, R., Kamtekar, S., Marx, K. A., Tripathy, S. K., Gao, H., et al. (1995). Molecular assembly of proteins and conjugated polymers: Toward the development of biosensors. Biotechnol. Bioeng. 45, 116-121. doi: 10.1002/bit. 260450204

Barth, J., Bergner, S. V., Jaeger, D., Niehues, A., Schulze, S., Scholz, M., et al. (2014). The interplay of light and oxygen in the reactive oxygen stress response of Chlamydomonas reinhardtii dissected by quantitative mass spectrometry. Mol. Cell. Proteomics 13, 969-989. doi: 10.1074/mcp.M113.032771

Beer, S., and Eshel, A. (1985). Determining phycoerythrin and phycocyanin concentrations in aqueous crude extracts of the red alga. Australi. J. Mar. Freshw. Res. 36, 785-792. doi: 10.1071/mf9850785

Benavides, J., and Rito-Palomares, M. (2004). Bioprocess intensification: a potential aqueous two-phase process for the primary recovery of B-phycoerythrin from Porphyridium cruentum. J. Chromatogr B Analyt. Technol. Biomed. Life Sci. 807, 33-38. doi: 10.1016/j.jchromb.2004.01.028

Bermejo, R., Acién, F. G., Ibáñez, M. J., Fernández, J. M., Molina, E., and Alvarez-Pez, J. M. (2003). Preparative purification of B-phycoerythrin from the microalga Porphyridium cruentum by expanded-bed adsorption light intensity and temperature, and the low inoculation resulted in the low biomass, PE, and EPS contents. In addition, the results of this study indicated that the proper extraction and purification method made a significant contribution to the high yield and purity of PE. Therefore, an integrated process was proposed to produce biomass and active compounds (B-PE and EPS) from photosynthetic microalga P. cruentum.

\section{DATA AVAILABILITY STATEMENT}

The original contributions presented in the study are included in the article/supplementary material, further inquiries can be directed to the corresponding author/s.

\section{AUTHOR CONTRIBUTIONS}

HW and T-ZL conceptualized the study. H-CY, J-KS, and T-LH validated the study. H-CY contributed to the manuscript writing and prepared the original draft of the manuscript. J-KS contributed to the manuscript writing and reviewed and edited the manuscript. All authors contributed to the article and approved the submitted version.

\section{FUNDING}

We would like to thank the National Key R\&D Program of China (Grant No. 2018YFA0902503), Shangdong Taishan Scholars Program (No. tsqn202103144), and the Innovation Foundation of QIBEBT (Grant: QIBEBT I201920) CAS.

chromatography. J. Chromatogr B Analyt. Technol. Biomed. Life Sci 790, 317-325.

Bermejo Román, R., Alvárez-Pez, J. M., Acién Fernández, F. G., and Molina Grima, E. (2002). Recovery of pure B-phycoerythrin from the microalga Porphyridium cruentum. J. Biotechnol. 93, 73-85. doi: 10.1016/s0168-1656(01)00 385-6

Bernaerts, T., Gheysen, L., Kyomugasho, C., Kermani, Z. J., and Loey, A. (2018). Comparison of microalgal biomasses as functional food ingredients: Focus on the composition of cell wall-related polysaccharides. Algal Res. 32, 150-161.

Breccia, D., Siñeriz, F., Baigori, D., and Castro, G. R. (1998). Purification and characterization of a thermostable xylanase from Bacillus amyloliquefaciens. Enzyme Microb. Technol. 22, 42-49. doi: 10.1007/s13205-017-0615-y

Bueno, M., Gallego, R., and Chourio, A. M. (2020). Green ultra-high pressure extraction of bioactive compounds from Haematococcus Pluvialis and Porphyridium cruentum microalgae[J]. Innov. Food Sci. Emerg. Technol. 66:102532. doi: 10.1016/j.ifset.2020.102532

Chopin, T., Yarish, C., Wilkes, R., Belyea, E., Lu, S., and Mathieson, A. J. J. (2000). Developing Porphyra/salmon integrated aquaculture for bioremediation and diversification of the aquaculture industry. J. Appl. Phycol. 12, 99-99.

Coward, T., Fuentes-Grunewald, C., Llewellyn, G., Lovitt, R. W., and Silkina, A. (2016). Utilising light-emitting diodes of specific narrow wavelengths for the optimization and co-production of multiple high-value compounds in Porphyridium purpureum. Bioresour. Technol. 221, 607-615. doi: 10.1016/j. biortech.2016.09.093

Dodgson, K., and Price, R. G. (1962). A note on the determination of the ester sulfate content of sulfated polysaccharides. Biochem. J. 84, 106-110. doi: 10. 1042/bj0840106 
Dubois, M., Gilles, K. A., Hamilton, J. K., Rebers, P. T, and Smith, F. (1956). Colorimetric method for determination of sugars and related substances. Anal. Chem. 28, 350-356. doi: 10.1021/ac60111a017

Dvir, I., Chayoth, R., Sod-Moriah, U., Shany, S., Nyska, A., Stark, A. H., et al. (2000). Soluble polysaccharide and biomass of red microalga Porphyridium sp. alter intestinal morphology and reduce serum cholesterol in rats. Br. J. Nutr. 84, 469-476.

Francisco, E. C., Neves, D. B., Jacob-Lopes, E., and Franco, T. T. (2010). Microalgae as feedstock for biodiesel production: carbon dioxide sequestration, lipid production, and biofuel quality. Chem. Technol. Biotechnol. 85, 395-403. doi: 10.1016/j.jphotobiol.2018.01.003

Fuentes, M., Fernández, G. G. A., and Pérez, J. A. (2000). Biomass nutrient profiles of the microalga Porphyridium cruentum. Food Chem. 70, 345-353. doi: 10. 1007/s00449-016-1676-8

Galland-Irmouli, A. V., Pons, L., Lucon, M., Villaume, C., Mrabet, N. T., Guéant, J. L., et al. (2000). One-step purification of R-phycoerythrin from the red macroalga Palmaria palmata using preparative polyacrylamide gel electrophoresis. J. Chromatogr B Biomed. Sci. Appl. 739, 117-123. doi: 10.1016/ s0378-4347(99)00433-8

Glazer, A. N. (1984). Phycobilisome a macromolecular complex optimized for light energy transfer. Biochim. Biophys. Acta 768, 29-51. doi: 10.1016/0304-4173(84) 90006-5

Gonzalez-Ramirez, E., Andujar-Sanchez, M., and Ortiz-Salmeron, E. (2014). Thermal and $\mathrm{pH}$ Stability of the B-Phycoerythrin from the Red Algae Porphyridium cruentum. Food Biophys. 9, 184-192. doi: 10.1007/s11483-0149331-x

Gough, S. P., and Kannangara, C. G. (1979). Biosynthesis of $\Delta$-aminolevulinate in greening barley leaves III: The formation of $\Delta$-aminolevulinate in Tigrigna mutants of barley. Carlsberg Res. Commun. 44, 403-416. doi: 10.1007/ bf02906189

Guillard, R. R., and Ryther, J. H. (1962). Studies of marine planktonic diatoms: i. Cyclotella nana hustedt, and detonula confervacea (cleve) gran. Can. J. Microbiol. 8, 229-239. doi: 10.1139/m62-029

Hilditch, C., Balding, P., Jenkins, R., Smith, A., and Rogers, L. J. (1991). R-phycoerythrin from the macroalgaCorallina Officinalis (Rhodophyceae) and application of a derived phycofluor probe for detecting sugar-binding sites on cell membranes. J. Appl. Phycol. 3, 345-354. doi: 10.1007/bf0239 2888

Hu, H., Wang, H. F., Ma, L. L., Shen, X. F., and Zeng, R. J. (2018). Effects of nitrogen and phosphorous stress on the formation of high-value LC-PUFAs in Porphyridium cruentum. Appl. Microbiol. Biotechnol. 102, 5763-5773. doi: 10.1007/s00253-018-8943-3

John, R. P., Anisha, G. S., Nampoothiri, K. M., and Pandey, A. (2011). Micro, and macroalgal biomass: A renewable source for bioethanol. Bioresour. Technol. 102, 186-193. doi: 10.1016/j.biortech.2010.06.139

Jones, R. F., Speer, H. L., and Kury, W. (2010). Studies on the Growth of the Red Alga Porphyridium cruentum. Physiol. Plant. 16, 636-643. doi: 10.1104/pp.91. 3.1179

Koller, K. P., Wehrmeyer, W., and Schneider, H. (1977). Isolation, and characterization of disc-shaped phycobilisomes from the red alga Rhodella violacea. Arch. Microbiol. 12, 61-67. doi: 10.1007/BF00446655

Lalibertè, G., and Noüie, J. D. L (2010). Auto-, Hetero-, And Mixotrophic Growth Of Chlamydomonas Humicola (Cmloroimiyckak) On Acetate. J. Phycol. 29, 612-620.

Li, Y., Horsman, M., Wu, N., Lan, C. Q., and Calero, N. D. (2008). Biofuels from microalga. Biotechnol. Pro. 24, 815-820.

Liu, T., Wang, J., Hu, Q., Cheng, P., Bei, J., Liu, J., et al. (2013). Attached cultivation technology of microalga for efficient biomass feedstock production. Bioresour. Technol. 127, 216-222. doi: 10.1016/j.biortech.2012.09.100

Maccoll, R. (1991). Fluorescence studies on R-phycoerythrin and C-phycoerythrin. J. Fluoresc. 1, 135-140.

Parmar, A., Singh, K. N., Kaushal, A., Madamwar, D. (2011). Characterization of intact phycoerythrin and its cleaved $14 \mathrm{kDa}$ functional subunit from marine cyanobacterium Phormidium sp. A27DM. Process Biochem. 46, 1793-1799.

Markou, G., and Georgakakis, D. (2011). Cultivation of filamentous cyanobacteria (blue-green alga) in agro-industrial wastes and wastewaters: a review. Appl. Energy 88, 3389-3401. doi: 10.1016/j.apenergy.2010.12.042

Marsac, N. (2003). Phycobiliproteins and phycobilisomes: the early observations. Photosynth. Res. 76, 193. doi: 10.1023/A:1024954911473
Mittal, R., Tavanandi, H. A., Mantri, V. A., and Raghavarao, K. (2017). Ultrasoundassisted methods for enhanced extraction of Phycobiliproteins from marine macro-alga. Ultrason. Sonochem. 38, 92. doi: 10.1016/j.ultsonch.2017.02.030

Papageorgiou, G. C., Tsimilli-Michael, M., and Stamatakis, K. (2007). The fast and slow kinetics of chlorophyll a fluorescence induction in plants, alga, and cyanobacteria: a viewpoint. Photosynth. Res. 94, 275-290. doi: 10.1007/s11120007-9193-x

Qiu, J., Madoz-Gurpide, J., Misek, D. E., Kuick, R., Brenner, D. E., Michailidis, G., et al. (2004). Development of natural protein microarrays for diagnosing cancer-based on antibody response to tumor antigens. J. Proteome Res. 3, 261-267. doi: 10.1021/pr049971u

Razaghi, A., Godhe, A., and Albers, E. (2014). Effects of nitrogen on growth and carbohydrate formation in Porphyridium cruentum. Cent. Eur J. Biol. 9, 156-162. doi: 10.2478/s11535-013-0248-z

Rizzo, R. F., Santos, B., Castro, G. F. P., Passos, T. S., Nascimento, M., Guerra, H. D., et al. (2015). Production of phycobiliproteins by Arthrospira platensis under different light conditions for application in food products. Food Sci. Technol. 35, 247-252. doi: 10.1590/1678-457x.6463

Romána, R. B., Alvárez-Pez, J. M., Acién Fernándezc, F. G., and Grimac, E. M. (2002). Recovery of pure B-phycoerythrin from the microalga Porphyridium cruentum. J. Biotechnol. 93, 73-85. doi: 10.1016/s0168-1656(01)00385-6

Schagger, H., and Jagow, G. V. (1987). Tricine-sodium dodecyl sulfatepolyacrylamide gel electrophoresis for the separation of proteins in the range from 1 to $100 \mathrm{kDa}$. Anal. Biochem. 166, 368-379. doi: 10.1016/0003-2697(87) 90587-2

Spolaore, P., Joannis-Cassan, C., Duran, E., and Isambert, A. (2006). Commercial applications of microalga. J. Biosci. Bioeng. 101, 87-96.

Srirangan, S., Sauer, M. L., Howard, B., Dvora, M., Dums, J., Backman, P., et al. (2015). Interaction of Temperature and Photoperiod Increases Growth and Oil Content in the Marine Microalga Dunaliella Viridis. PLoS One 10:e0127562. doi: 10.1371/journal.pone.0127562

Sun, L., Wang, C., Shi, Q., and Ma, C. (2009). Preparation of different molecular weight polysaccharides from Porphyridium cruentum and their antioxidant activities. Int. J. Biol. Macromol. 45, 42-47. doi: 10.1016/j.ijbiomac.2009.03.013

Talyshinsky, M. M., Souprun, Y. Y., and Huleihel, M. M. (2002). Anti-viral activity of red microalgal polysaccharides against retroviruses. Cancer Cell Int. 2:8. doi: 10.1186/1475-2867-2-8

Vonshak, A. (1988). "Porphyridium," in Microalgal Biotechnology, eds, M. A. Borowitzka, and L. J. Borowitzka (Cambridge: Cambridge University Press), $122-134$.

Zhang, J., Ma, J., Liu, D., Qin, S., Sun, S., Zhao, J., et al. (2017). Structure of phycobilisome from the red alga Griffithsia Pacifica. Nature 551, 57-63. doi: 10.1038 /nature 24278

Zhang, Z. U. (2003). Saccharide Complex Biochemical Research Technology, 2nd edtion, China: Zhejiang University, 11-16.

Zheng, H., Yin, J., Gao, Z., Huang, H., Ji, X., and Dou, C. J. A. B. (2011). Biotechnology, Disruption of Chlorella Vulgaris Cells for the Release of Biodiesel-Producing Lipids: A Comparison of Grinding, Ultrasonication, Bead Milling, Enzymatic Lysis, and Microwaves. Appl. Biochem. Biotechnol. 164, 1215-1224. doi: 10.1007/s12010-011-9207-1

Zhu, L., Yan, C., and Li, Z. (2016). Microalgal cultivation with biogas slurry for biofuel production. Bioresour. Technol. 220, 629-636 doi: 10.1016/j.biortech. 2016.08.111

Conflict of Interest: The authors declare that the research was conducted in the absence of any commercial or financial relationships that could be construed as a potential conflict of interest.

Publisher's Note: All claims expressed in this article are solely those of the authors and do not necessarily represent those of their affiliated organizations, or those of the publisher, the editors and the reviewers. Any product that may be evaluated in this article, or claim that may be made by its manufacturer, is not guaranteed or endorsed by the publisher.

Copyright (c) 2022 Yin, Sui, Han, Liu and Wang. This is an open-access article distributed under the terms of the Creative Commons Attribution License (CC BY). The use, distribution or reproduction in other forums is permitted, provided the original author(s) and the copyright owner(s) are credited and that the original publication in this journal is cited, in accordance with accepted academic practice. No use, distribution or reproduction is permitted which does not comply with these terms. 